\title{
Experiencia religiosa en ambientes virtuales de aprendizaje.
}

\author{
Yefren Díaz López ${ }^{1}$ \\ Hna. Delia Patricia Gómez Salazar ${ }^{2}$
}

\begin{abstract}
Resumen
El artículo es resultado del proyecto de investigación «Cartografía social de la experiencia religiosa en los ambientes virtuales de aprendizaje», que desde una perspectiva analítica, aborda el mapeo de los espacios virtuales de aprendizaje, que a su vez, son referentes ciberespaciales en los cuales se construye la red-comunidad de aprendizaje, y se expresan las múltiples vivencias humanas y religiosas, desde el estilo cristiano de presencia en el mundo digital. El análisis fue orientado desde el procedimiento metodológico teológico (texto, contexto, pretexto), se utilizó la técnica de la cartografía social, así como las herramientas e instrumentos tecnológicos propios de los ambientes virtuales de aprendizaje, y los mapas colectivos de las experiencias humanas y religiosas, en el espacio y la red-comunidad virtual de aprendizaje de la Licenciatura en Ciencias Religiosas, Facultad de Teología. Pontificia Universidad Javeriana.
\end{abstract}

Palabras clave: ambientes virtuales de aprendizaje, cartografía social, contexto educativo, experiencia religiosa, tecnologías de la información. 


\title{
Religious experience in virtual learning environments.
}

\begin{abstract}
This paper is the result of the research project "Social Mapping of Religious Experience in Virtual Learning Environments". From an analytical perspective, addresses the mapping of virtual learning spaces, which are cyberspace referents in which a network-learning community is built. Many human and religious experiences are expressed, from the Christian style in the digital world. This is analysis directed from the theological methodological approach (text, context, and pretext), the Social Cartography technique, tools and technological instruments specific to virtual learning environments, and collective maps of human and religious experiences in the network-learning community of la BSc in Religion Sciences. Faculty of the Theology. Pontificia Universidad Javeriana.
\end{abstract}

Key words: Environments, Virtual, Learning, Experience, Religious.

Recibido: 24 de julio de 2013 Aceptado: 05 de noviembre de 2013

\section{lntroducción}

Con el Siglo XXI, la internet, ha dado origen a nuevos espacios de interacción y comunicación, que se encuentran referenciados en lo que hoy se llama ciberespacio, entendido como «espacio de comunicación abierto por la interconexión mundial de los ordenadores y de las memorias informáticas» (Levy, 2007: 70). El contexto educativo, enmarcado por la realidad del ciberespacio, aparece como una nueva realidad que está revolucionando la historia educativa, es la llamada educación virtual. Unigarro expresa la incidencia educativa dada en el ciberespacios, por el cual se da una fuerte transformación.

Se genera un proceso educativo, una acción comunicativa con intenciones de formación, en un lugar distinto al salón de clases: en el ciberespacio; en una temporalidad que puede ser sincrónica o asincrónica y sin la necesidad de que los cuerpos de maestros y alumnos estén presentes (Unigarro, 2004: 46). 
Para el caso de la comunidad virtual de la Licenciatura en Ciencias Religiosas (LCRV), esta nueva realidad está determinando, en cada uno de los sujetos, una manera de ocupar los espacios virtuales, unos estilos de comportamiento en la interacción entre realidad virtual y realidad presencial, y unas nuevas formas de ser, por lo que Yenes afirma: «la forma de funcionar basada en las tecnologías de la información» (2000: 240).

De esta manera, dentro de los antecedentes que sustentan el presente artículo, se encuentra la investigación: Cartografía Social de la Experiencia Religiosa en los Ambientes Virtuales de Aprendizaje AVA, la cual se desarrolló desde el diseño de Investigación Acción Participativa (IAP), entendida con Fals Borda como aquella que: "más allá de la observación es participación, acción y estudio. Donde hay que estar dentro de la comunidad y participar de sus objetivos de cambio social" ; y los aportes de la hermenéutica teológica latinoamericana y del Caribe, que en lo expresado por Parra (2003: 18), articula metodológicamente "el texto de tradición, el contexto de situación y el propósito redentor y liberador".

Siendo de interés el tema de la Experiencia Religiosa en los AVA en cuanto a lo que expresa Parra (2003: 198) la incidencia de un proceso reflexivo desde la teología, ha de darse desde la praxis histórica de los hombres, que en el caso de la praxis histórica de la comunidad virtual de aprendizaje, se expresa a través de las vivencias humanas y religiosas de protestantes y católicos.

El objetivo de la reflexión pretende referenciar el impacto de la Experiencia Religiosa en los espacios de la Comunidad Virtual de Aprendizaje de la Licenciatura en Ciencias Religiosas, Facultad de Teología. Pontificia Universidad Javeriana (LCRV); acorde con las políticas de la PUJ y la Facultad de Teología, que busca formar personas de manera integral. Desde el análisis temático que fundamenta los resultados de la investigación se evidencia que se puede ser tan humano en la red como fuera de ella, concluyendo que si la base de la experiencia religiosa es la experiencia humana, entonces la experiencia religiosa que se vive en los AVA es real.

\section{Planteamiento del problema}

En este trabajo se parte de la realidad expresada por Gazzola y Didriksson, sobre América Latina y el Caribe, según la cual "se encuentra determinada por su ubicación de exclusión en el marco de las brechas y asimetrías en las que ocurre la división internacional de los conocimientos, de la innovación tecnológica y de la revolución de la ciencia y de sus aplicaciones" (2008:25). 
Realidad a la que sumamos los cambios propios del nuevo milenio, como la "sociedad del conocimiento y la información", entendida como aquella en la que las tecnologías, facilitan la creación, distribución y manipulación de la información jugando un papel importante en las actividades sociales, culturales, económicas y educativas. Por lo que expresa Unigarro que "uno de los retos principales, si no el más importante de los que debe abordar la educación de nuestros días, se refiere a la responsabilidad que tiene frente al conocimiento y a la nueva manera de concebirlo" (2004: 46).

De esta manera se busca, a través del mapeo de los espacios virtuales de aprendizaje, la representación de mapas colectivos y el análisis de contenido de la descripción de las experiencias humanas y religiosas propias de los integrantes de la comunidad virtual de aprendizaje, así como delimitar los referentes espaciales que respondan a la pregunta ¿Cuál es la Experiencia Religiosa de la Comunidad Virtual de Aprendizaje de la LCRV de la PUJ?; desde el objetivo que busca reconocer en la Licenciatura en Ciencias Religiosas los Ambientes Virtuales de Aprendizaje, como contextos reales de interacción subjetiva e intersubjetiva, en los cuales se están dando nuevos tipos de vivencias humanas y experiencias religiosas.

\section{Desarrollo del tema}

\section{Antecedentes}

El contexto en el que se ubica esta investigación, estuvo orientado por el Ámbito de la misión «ad gentes», la cual en virtud del mandato universal de Cristo no conoce confines, resaltando la importancia de los ámbitos territoriales. En esta misma perspectiva recuerda la Redemptoris missio (1990) que: "la actividad misionera ha sido definida normalmente en relación con territorios concretos" Cap, IV Nro. 37, lo que permite inferir, que de igual forma se debe irrumpir en los nuevos ambientes virtuales, como espacios reales de interacción. En el marco de los límites geográficos, la Redemptoris missio añade:

“...no puede aceptar que límites geográficos o dificultades de indole política sean obstáculo para su presencia misionera, de la misma manera, tampoco podrá mantenerse al margen de los nuevos límites presencialesvirtuales que se tratan de mantener, para poder así responder a los retos de su actividad misionera ad gentes, en los nuevos espacios virtuales ocupados por los diferentes grupos humanos. De ahi que, el criterio geográfico, aunque no muy preciso y siempre provisional, sigue siendo válido todavía para indicar las fronteras hacia las que debe dirigirse la actividad misionera" (No 37). 
Y aunque apenas la Iglesia se está aproximando a los contextos virtuales, al reconocer que no basta usar los medios de comunicación social, debe ir más allá de su reconocimiento, hacia la integración de su valor al servicio del desarrollo de la comunicación. A este fin lo expresa el documento eclesial Aetatis Novae:

“... conviene integrar el mensaje mismo en esta "nueva cultura" creada por la comunicación moderna... con nuevos lenguajes, nuevas técnicas, nuevos comportamientos sicológicos” (Capítulo IV, No. 18).

A estos antecedentes se suma el estudio de caso en la experiencia del proceso de virtualización de la LCRV realizado en el 2009. Lo que llevó a retomar desde Levy (2010) (citado en el estudio de caso de la PUJ por Castillón, Bermúdez, Díaz y García, 2009: 3) el concepto de ciberespacio entendido como: "el oceánico universo de informaciones que contiene, así como los seres humanos que navegan por él y lo alimentan; y Cibercultura como un conjunto de técnicas, por lo que se refiere a materiales educativos computarizados a las producciones intelectuales, las prácticas, las actitudes, los modos de pensamiento y los valores que se desarrollan conjuntamente en el crecimiento del ciberespacio".

\title{
Incorporación de las TIC en los ambientes de aprendizaje
}

En una sociedad cambiante y un mundo globalizado que no se detiene, donde la tecnología, la cultura, y la ciencia evolucionan a cada instante, se plantea la necesidad de una educación significativa para los estudiantes, que facilite su desarrollo integral, desdes la articulación al contexto educativo, enmarcado por la realidad del ciberespacio, de la nueva realidad que está revolucionando la historia educativa, la llamada educación virtual; a este fin expresa Unigarro:

\begin{abstract}
Que genera un proceso educativo, una acción comunicativa con intenciones de formación, en un lugar distinto al salón de clases: en el ciberespacio; en una temporalidad que puede ser sincrónica o asincrónica y sin la necesidad de que los cuerpos de maestros y alumnos estén presentes... acción que busca propiciar espacios de formación de los sujetos y que, apoyándose en las tecnologías de la información y la comunicación, instaura una nueva manera de establecer encuentro comunicativo entre los actores del proceso (1994: 46).
\end{abstract}

Por lo tanto, en la actual época de profundas transformaciones y cambios que afectan irreversiblemente al conjunto de la humanidad, no es posible responder a la realidad desde la educación tradicional surgida por la revolución industrial; es preciso ahora, pensar la educación desde la nueva sociedad del conocimiento. 
Razón por la cual en la universidad el uso de las TIC está incursionando con nuevas formas de construir conocimiento, por ejemplo: frente al trabajo docente resulta un reto y una tarea formar parte de los avances tecnológicos y hacer de ellos un recurso para llevar a los estudiantes el conocimiento; los grupos académicos permiten la creación de comunidades virtuales que emplean los recursos que ofrece la red; y los procesos investigativos son compartidos y enriquecidos con personas que se encuentran en diferentes espacios y contextos culturales a través del uso de las herramientas tecnológicas.

De esta manera, desde la experiencia que en cada contexto se puede dar de un proceso investigativo, como lo expresa el documento Columbus: "han surgido nuevas tecnologías que constituyen un reto para los métodos de docencia e investigación (...) e incluso, la definición misma de la misión de la universidad y del rol que ésta desempeña en la sociedad" (1987: 5).

En consecuencia, la incorporación de las TIC a los ambientes de aprendizaje evidencia la necesidad de adecuar la educación al contexto de la sociedad del conocimiento y la información, siendo conscientes de su obligación de preparar a las personas para desarrollarse integralmente en la sociedad que viven. Donde los multicontextos virtuales no son entendidos como simulación de lo real, en cuanto permiten la interacción de personas reales, desde sus contextos reales.

Ante el gran desafío de concebir una nueva pedagogía apoyada en los medios tecnológicos y la capacidad de trascendencia en aras al desarrollo de un conocimiento que responda a la realidad, como necesidad del contexto local y global, la educación virtual tiene hoy mucho por decir ya que abre posibilidades de acercamiento intersubjetivo.

La forma más moderna y más potente de ofrecer información y con ella, recursos para la educación, es la World Wide Web. De esto modo, la web es el resultado de la interconexión de miles de computadoras de todo el mundo. Todas ellas comparten los protocolos de comunicación, es decir, todas hacen uso de la misma tecnología para establecer una comunicación a través de ellas. De igual forma, se establecen espacios para compartir información, en los que de forma abierta cualquier usuario puede acceder y en los que de forma privada, se requiere contar con los requisitos de acceso.

Estos nuevos contextos virtuales no son entendidos como simulación de lo real, y aunque el ciberespacio que está siendo producido por una interfaz (objetos tangibles, esto es, cables, computadoras, hardware, software, etc.), no es en sí mismo físico o tangible, sí permite la interacción de personas reales desde sus contextos reales.

En este contexto real del ciberespacio, se encuentra la comunidad virtual de aprendizaje, determinada, como lo plantea Levy (citado en el estudio de caso 
de la PUJ), por el deseo de comunicación recíproca de quienes acceden a él, y que tiene como finalidad la construcción de una inteligencia colectiva que permita generar una construcción común del conocimiento.

Conocimiento colectivo que en la LCRV se construye en los ambientes virtuales de aprendizaje que se configuran en lugar de encuentro para el aprendizaje autónomo y colaborativo, propio de la inteligencia colectiva, con el fin de socializar y democratizar dicho saber en el mundo de hoy por medio de las redes virtuales. De esta manera, Cadillo León define la comunidad virtual como aquella comunidad cuyos vínculos, interacciones y relaciones tienen lugar no en un espacio físico sino en un espacio virtual como internet. Y autores como Mas, et al. , comprenden que esto exige «...nuevos compromisos, nuevas formas de percibir la relación con el otro, teniendo presente, que el proceso dialógico es multidireccional» (2006: 1$)$.

Por tanto, los medios de comunicación y la tecnología no pueden considerarse como algo frío o deshumanizante, Bach y Fores creen que «pueden llegar a ser tan humanos, tan cálidos, tan formativos, como capacidad tengamos para generar en ellos (...) monumentos de comunicación, encuentro o crecimiento» (2007: 28). Por ello, la necesidad de evidenciar que se es tan humano en la red como fuera de ella.

\section{La experiencia humana como base de la experiencia religiosa}

Si como afirman Bach y Fores : «Realidad y virtualidad no tienen por qué ser dos mundos contrapuestos e incompatibles. No es cierto que el primero garantice un mayor acercamiento entre los seres humanos, así como una vivencia personal más rica y directa, y que el segundo sea frío, desnaturalizado o distante» (2007: 28). Razón por la cual se entiende la experiencia humana como la define Croatto «una vivencia relacional, de manera diversificada", que se manifiesta en diferentes espacios como: el mundo, el otro individual y el grupo (...), reconociendo que «sobre la base de la vivencia humana, o mejor en sus fibras más íntimas, se inserta la experiencia religiosa» (2002: 40).

Por tanto, las experiencias humanas tienen un alcance no sólo experiencial, sino también revelador, en cuanto afirma Croatto, «El ser humano ha sabido en todos los tiempos "imaginar" modos de superar sus límites mediante el recurso de lo sagrado», por lo que el Concilio Vaticano II en el documento Dei Verbum (1965) confiesa que:

Dios, principio y fin de todas las cosas, puede ser conocido con seguridad por la luz natural de la razón humana, partiendo de las criaturas" (cf. Rom., 1, 20); pero enseña que hay que atribuir a su revelación "el que todos, aun en la presente condición del género humano, puedan conocer fácilmente, con firme certeza y sin ningún error, las cosas divinas que por su naturaleza no son inaccesibles a la razón humana" (No. 7). 
A partir de las experiencias humanas, Hungs reconoce que «la experiencia religiosa nunca es algo cerrado en sí mismo, (...) sino que se constituye en relación con otras personas» (1982: 109), es así como para Zanotto la experiencia en clave religiosa que constituye una experiencia reveladora de Dios, también es «... lectura en profundidad de lo vivido, hasta el nivel del misterio y la apertura al trascedente» (2006: 152).

Esto motiva a volver la mirada sobre el papel de la teología en el desarrollo de la reflexión de Dios y la praxis de la experiencia que esto suscita en el ser humano, convirtiéndose en tarea dentro de la reflexión en materia de teología. A este fin, Gibellini , afirma que:

La teología está llamada a mantener abierta la comunicación entre los contenidos tradicionales de la fe y la experiencia humana, en una constante relación crítica entre ambas fuentes: la tradición bíblica y nuestro mundo actual (...) la historia transmitida de las experiencias originarias del pasado, tienen autoridad en la medida que revelan y comunican un sentido que pone en pie a los hombres y mujeres que afianza y libera (1998:366).

Esto nos permite comprender la experiencia religiosa más allá de una condición de fe, a lo que González (2008) señala como "un fenómeno amplio en el ser humano, que es la búsqueda de significado". La experiencia religiosa vista desde el lugar de la Revelación, como manifestación íntima de Dios al ser humano y conciencia que el mismo adquiere de esta presencia, señala una nueva postura ante su propia vida, como la de los demás. Zanotto (2006: 151) habla de esta revelación, como lugar de relectura de la historia, donde el ser humano alcanza conciencia de las personas y la presencia de Dios.

Velasco (1989: 82) habla de la incidencia de la comunión en la experiencia de encuentro que suscita la experiencia religiosa, caracterizada por el deseo de comunión con lo divino, que impulsa un proceso de maduración en el ser humano de los afectos y los deseos, por lo cual, la realidad percibida se torna buena y hermosa con la que el sujeto se siente en comunión. Comunión que da sentido a la existencia y la transforma, la hace mirar más allá de sí y salir al encuentro del totalmente Otro, en los otros y otras, asumiendo con Croato que "así como desde un punto de vista es individual y social, desde otro es una experiencia del mundo (lo que conduce a una cosmovisión), de Dios (teovisión) y del hombre (ética)" (2002: 62).

Es desde esta experiencia religiosa que la teología se puede aproximar a los AVA, reconociendo que no basta usar las herramientas tecnológicas o medios de comunicación social, sino, como lo expresa el documento eclesial Aetatis Novae : “... conviene integrar el mensaje mismo en esta "nueva cultura" 
creada por la comunicación moderna... con nuevos lenguajes, nuevas técnicas, nuevos comportamientos sicológicos" (Capítulo IV, No. 18), favoreciendo las vivencias humanas que se están dando en estos espacios como base de las experiencias religiosas que se deben acompañar.

\section{Referenciación de la experiencia religiosa en los AVA de la comunidad virtual de Aprendizaje de la LCRV de la PUJ}

El análisis temático hasta aquí realizado se fundamenta en los resultados de la investigación que evidencia el poder ser tan humano en la red como fuera de ella, de donde se sustenta, que si la base de la experiencia religiosa es la experiencia humana, entonces la experiencia religiosa que se vive en los AVA es real. Asumiendo la cibercultura y el ciberespacio como lugares propios de la sociedad del conocimiento y la información hacia el nuevo horizonte del contexto de situación de los y las intérpretes que conforman la comunidad virtual de aprendizaje, que desde los aportes de la hermenéutica teológica latinoamericana y del Caribe y lo expresado por Parra (2003: 18) articulan metodológicamente "el texto de tradición, el contexto de situación y el propósito redentor y liberador".

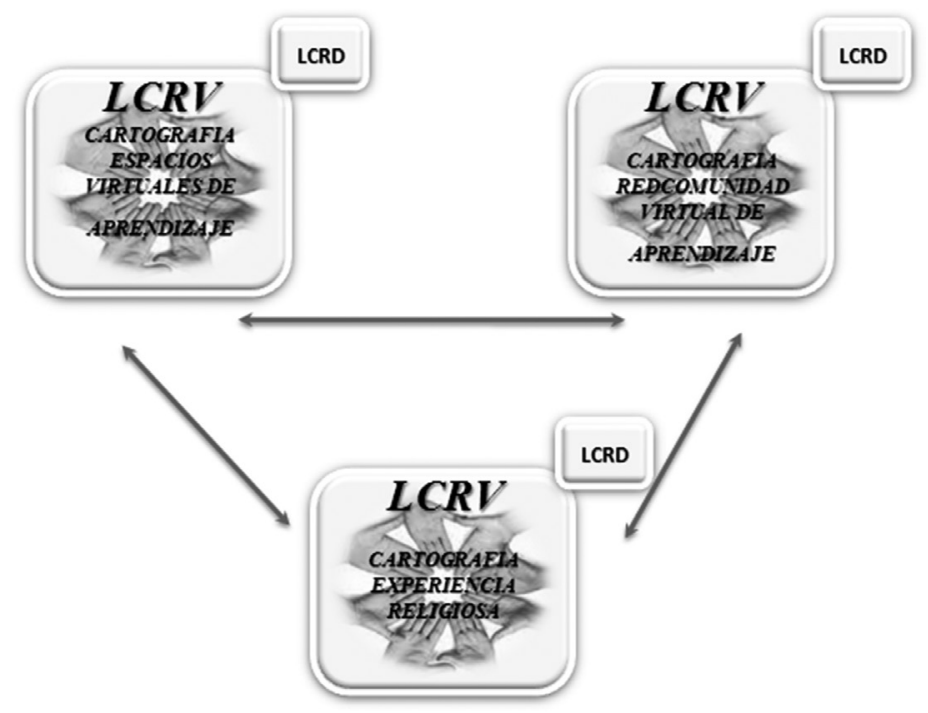

Fig. 1. Mapa Texto, Contexto y Pretexto de la Cartografía social de la Experiencia Religiosa de la comunidad virtual de Aprendizaje en los AVA. 


\section{Contexto: identificación y ocupación de los AVA en la LCRV}

Desde el acuerdo 461 dado en el plan de desarrollo de tecnología, la PUJ reconoce que "el Internet tiene el potencial de difundir y expandir el Aprendizaje a distancia como nunca antes". Llevando a identificar nuevos contextos de situación propios del programa como parte fundamental, aunque no suficiente, en el proceso de construcción del conocimiento, de enseñanza - aprendizaje y desarrollo humano de todos y cada uno de los integrantes de la comunidad virtual de aprendizaje:

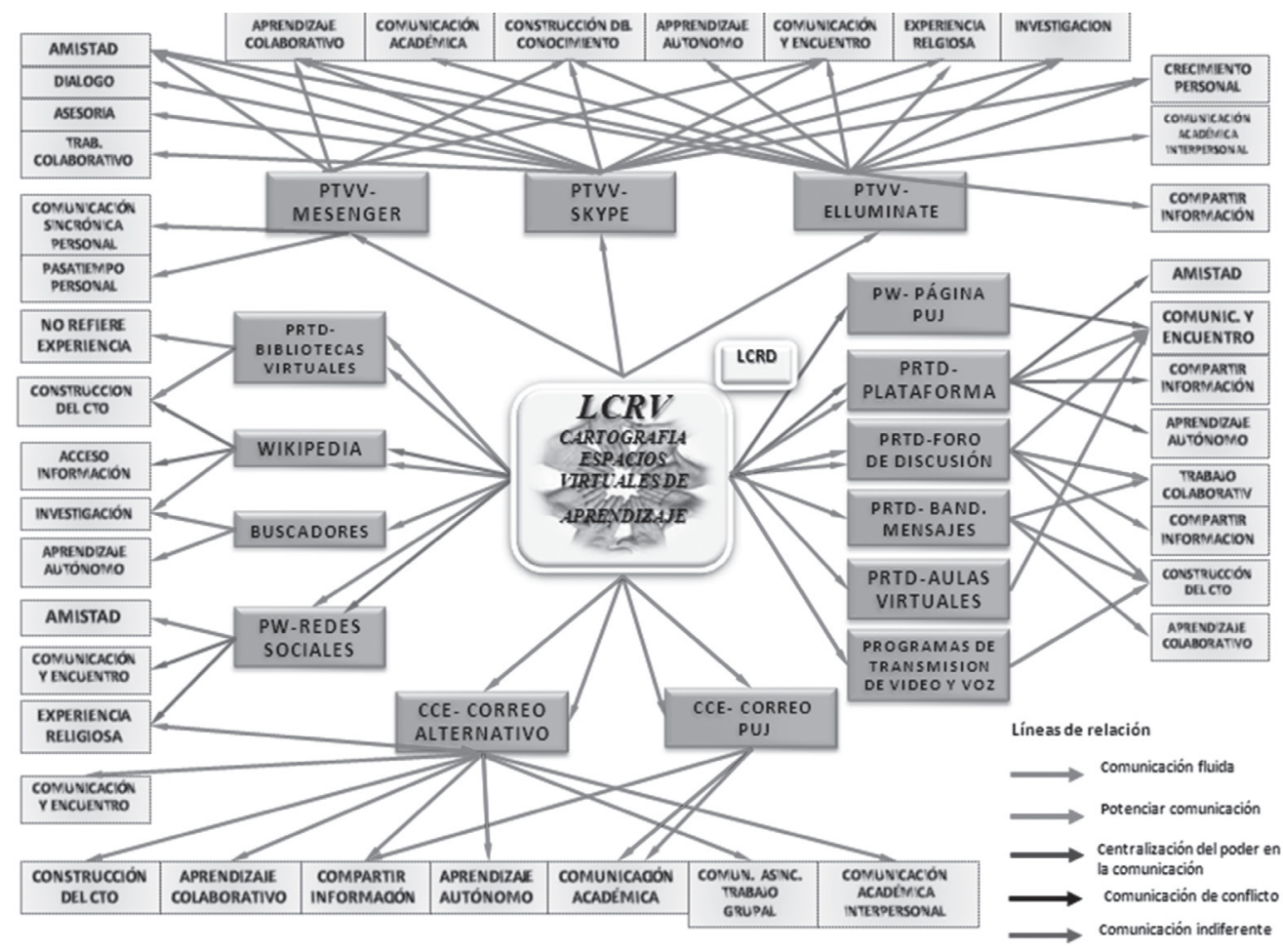

Fig. 2. Mapa Cartografía Social de los AVA. Experiencias que se dan en los espacios virtuales de aprendizaje por la comunidad virtual de aprendizaje LCRV. Realizado por María Isabel Torres, Natalia Andrea Laverde, Patricia Gómez y Ricardo García. Licenciados en Ciencias Religiosas-Facultad de Teología de la PUJ.

Los espacios virtuales sincrónicos y asincrónicos más reconocidos por la comunidad virtual como posibilitadores de encuentros humanos y religiosos son: Skype, calificado como el lugar más utilizado por hacer posible el aprendizaje colaborativo y las experiencias de comunicación fluida, amistad, y dialogo en torno a experiencias trascendentes significativas; Elluminate por facilitar la construcción de saberes y de experiencias de vida en torno a los aprendizajes teológicos propios del programa; y el Correo Electrónico 
institucional y alternativos (Hotmail, Yahoo, Gmail) donde la palabra escrita expresa otra forma de presencia tan real y efectiva como la palabra pronunciada, permitiendo el intercambio de conocimientos, experiencias personales y colectivas (humanas y religiosas).

\section{Texto: identificación de las experiencias humanas y religiosas en los espacios de la $L C R V$}

Partiendo de la dinámica interpretativa del método hermenéutico teológico, comprendemos desde la postura de Parra en el texto de las experiencias, la ocasión oportuna para generar un «proceso de interrogación y comprensión de las mismas en el horizonte de la revelación y la salvación» (2003: 229). Visibilizando a la comunidad virtual de aprendizaje como la autora del texto de experiencias tanto humanas como religiosas, que en términos comunitarios o colectivos reflejan, Parra «en la conciencia comunitaria y eclesial, su conciencia dogmática acerca de Dios, de su gracia, de su salvación, de su revelación» (2003: 230).

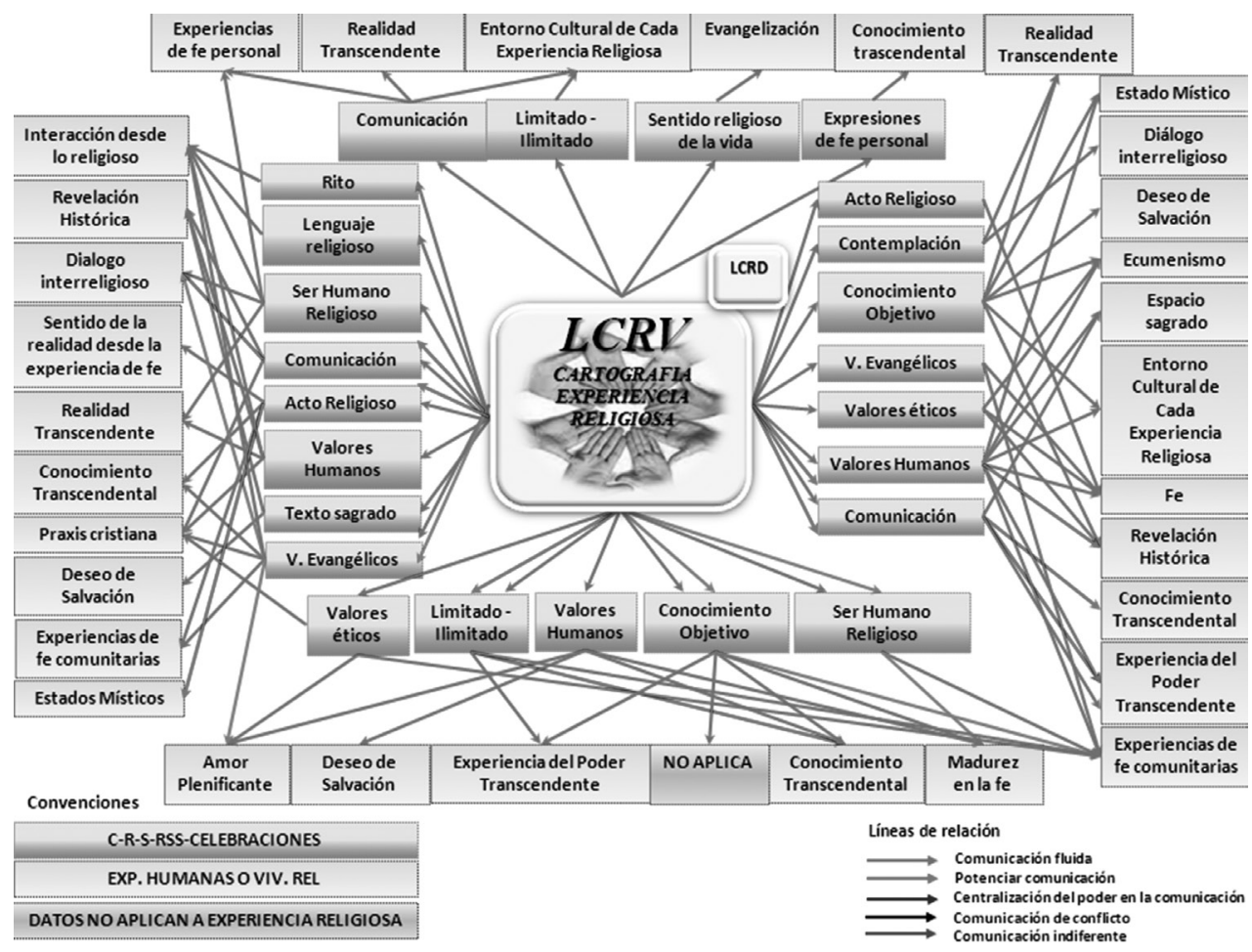

Fig. 3. Mapa síntesis de la cartografía social de la Experiencia Religiosa.Experiencias Religiosas que se dan en la interacción entre los miembros de la comunidad virtual de aprendizaje de la LCRV. Realizado por Marta Dávila, Enith Carreño, Martín Duque y Ramón Sabogal. 
En este mapa cartográfico de la experiencia religiosa de la comunidad virtual de aprendizaje, se puede apreciar el cúmulo de experiencias dadas en el encuentro intersubjetivo, dentro de las que sobresalen las experiencias de fe comunitaria, ecumenismo, deseo de salvación, conocimiento trascendental, madurez en la fe, praxis cristiana, como experiencias que se encuentran en profunda relación con los valores humanos referenciados preferentemente en los espacio propios de los encuentros sincrónicos como Skype, Elluminate Live y MSN.

Así, desde el texto de la experiencia religiosa cartografiado se resaltan los valores humanos como principios inherentes a las experiencias humanas dadas al interior de los (AVA), permitiendo que se trasciendan y se asuman desde el encuentro revelador de Dios que compromete a la persona que lo experimenta desde la praxis de los valores evangélicos.

\section{Pretexto: experiencias humanas y religiosas en los AVA de la LCRV}

Uno de los pretextos evidenciados en los mapas de la cartografía social, de la experiencia religiosa en los espacios y la red comunidad virtual de aprendizaje de la LCRV, es orientar la ocupación de los territorios propios del ciberespacio, demarcando como frontera los que favorecen el aprendizaje y el desarrollo humano, a través de la praxis ética de liberación que se evidencia en las experiencias humanas y religiosas.

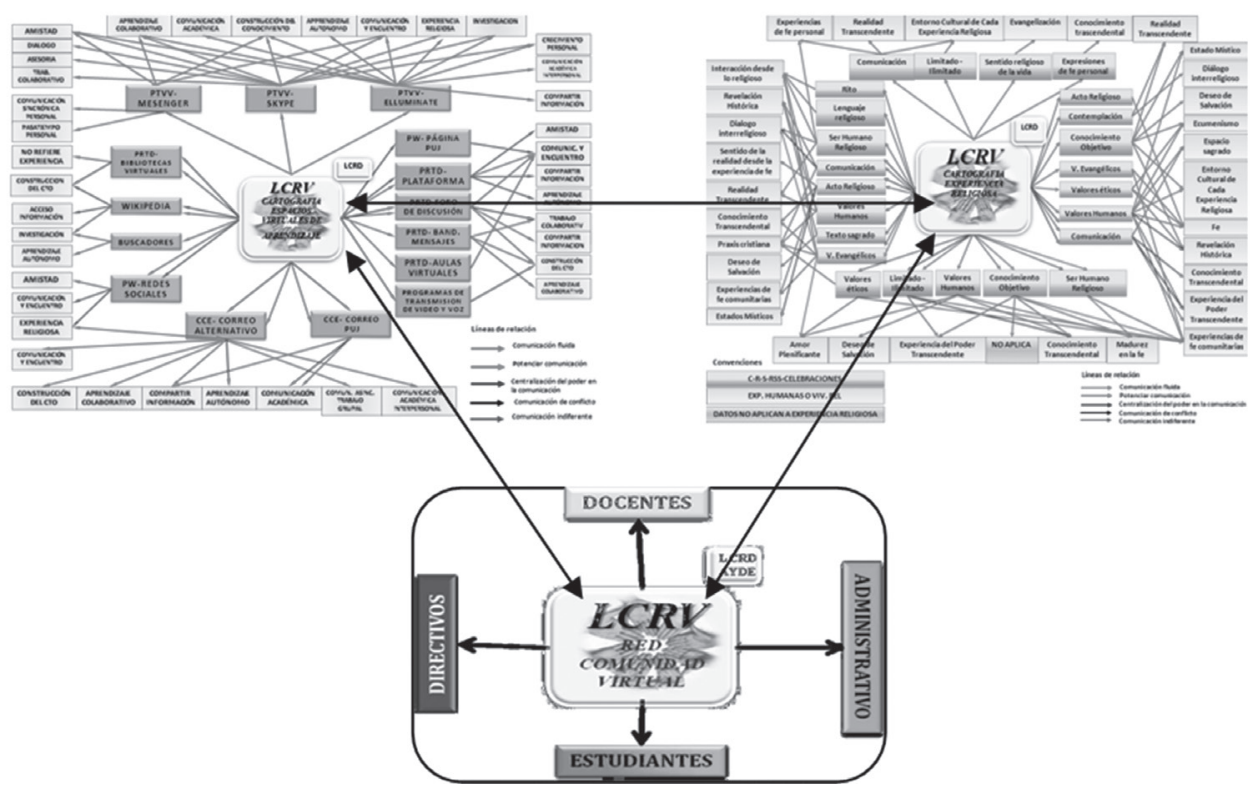

Fig. 4. Mapa síntesis de la cartografía social de la red comunidad virtual de aprendizaje. Interacción Experiencia religiosa de la Red comunidad virtual en los AVA. Realizado por Diana Orjuela, Claudia Peña, Rosanyela Buitrago. 
De esta manera, las personas que conforman los diferentes nodos de la Red comunidad virtual de aprendizaje, desde su identidad cristiana e interacciones que caracteriza, asumen como parte de sus pretextos las palabras del Papa Benedicto XVI en la $45^{\mathrm{a}}$ jornada mundial de las comunicaciones, donde pide a todos los cristianos que:

Cuando se intercambian informaciones, las personas se comparten a sí mismas, su visión del mundo, sus esperanzas, sus ideales. Por eso, puede decirse que existe un estilo cristiano de presencia también en el mundo digital, caracterizado por una comunicación franca y abierta, responsable y respetuosa del otro. Comunicar el Evangelio a través de los nuevos medios significa no sólo poner contenidos abiertamente religiosos en las plataformas de los diversos medios, sino también dar testimonio coherente en el propio perfil digital y en el modo de comunicar preferencias, opciones y juicios que sean profundamente concordes con el Evangelio, incluso cuando no se hable explícitamente de él.

\section{Conclusiones}

En la red comunidad virtual de aprendizaje, no es posible hablar de espacios académicos que formen a los estudiantes al margen de una reflexión alejada de la realidad que acontece en el los multicontextos presenciales y/o virtuales, de ahí, la necesidad de propuestas que orienten una praxis ético pedagógica y/o pastoral liberadora, comprometida con las transformación social de los territorios ocupados en el ciberespacio.

En el contexto ciberespacial, el pretexto que nos interpela es el rescatar la humanidad de los sujetos, que desde prácticas instrumentales, ocupan los nuevos multicontextos virtuales, a través del reconocimiento de la axiología manifiesta en sus experiencias, develando si las mismas favorecen el desarrollo como seres humanos o, por el contrario, los deshumaniza e instrumentaliza en aras de mantener nuevos tipos de opresión y esclavitud.

Asumir el reto de transformar e innovar las prácticas pedagógicas que se dan en la comunidad virtual de aprendizaje, desde la necesidad de proyectar el horizonte de los espacios académicos a la fundamentación de la propia praxis pedagógica cristiana, expresada en la vida de los miembros de la comunidad virtual como testimonio del paso de Dios en su existencia.

Fundamentar una postura académico-tecnológica donde se reconozcan los espacios virtuales de aprendizaje o los ambientes virtuales de aprendizaje, como contextos reales de interacción subjetiva e intersubjetiva, en los cuales se están dando un nuevo tipo de vivencias humanas y experiencias religiosas. 


\section{Referencias bibliográficas}

Bach, E y Fores, A. 2007. E-mociones: comunicar y educar a través de la red. Barcelona: Editores CEAC.

Cadillo León, J. s.f. Conceptos básicos de una comunidad virtual. Extraído el 5 de Abril de 2011 desde: http://es.scribd.com/doc/24987906/Conceptos-sobre-ComunidadesVirtuales

Castillón, J.F, Bermúdez, L., Díaz, Y, y García, D. 2009. Las tecnologías de la información y la comunicación en el horizonte de la cibercultura ante el reto de la formación integral en la Pontificia Universidad Javeriana. Estudio de caso en la experiencia del proceso de virtualización de la Licenciatura en Ciencias Religiosas Virtual. Investigación realizada, mediante Id de proyecto 2396 ante Vicerrectoría Académica, por el grupo de investigación de la Licenciatura en Ciencias Religiosas virtual, Facultad de Teología, Bogotá: Pontificia Universidad Javeriana.

Conciclio Vaticano. Constitución Dogmática Dei Verbum. Sobre la Divina Revelación. Nro. 7 Extraído el 10 de Abril de2011 desde: http://www.vatican.va/archive/hist councils/ii_vatican_council/documents/vat-ii_const_19651118_dei-verbum_sp.html

Croatto, J. 2002. Experiencia de lo sagrado y tradiciones religiosas. Navarra: Verbo Divino.

Gazzola. A.L. y Didriksson, A. 2008. Tendencias de la educación superior en América Latina y el Caribe. Instituto Internacional de la Unesco para la Educación Superior en América Latina y el Caribe. Caracas 2008. Cap. I. Extraído el 20 de Abril de 2011 desde: http://wwwisis.ufg.edu.sv/wwwisis/documentos/EB/378.121-T291/378.121T291-Tendencias\%20de\%2ola\%20educacion\%2osuperior\%20en\%20america\%2olatina\%20 y\%20el\%20caribe.pdf

Gibellini, R. 1998. La teología del siglo XX. Maliaño: Ediciones Sal Terrae. Extraído el 5 de Mayo desde: http://books.google.com/books?id=gcK2hXeLoaAC\&printsec=fron tcover\&dq=La+teolog\%C3\%ADa+del+siglo+XX\&hl=es\&ei=CIXjTaWOFISIrAfgmM3JBg $\& s a=X \& o i=b o o k \_r e s u l t \& c t=r e s u l t \& r e s n u m=1 \& v e d=0 C C k Q 6 A E w A A \# v=$ onepage $\& q \&$ $f=f a l s e$

Hungs, F.J. 1982. Comunidad y catequesis. Ediciones Sal Terrae. Pág. 109 Extraído el 5 de Abril de 2011 desde: http://books.google.com/books?id=M56nuGyX2XcC\&pg=PA7 $7 \& d q=$ Comunidad $+y+$ catequesis + de + Hungs\&hl=es\&ei=1YPjTZ2EC8TsrQfTylmrBg\&sa $=X \&$ oi $=$ book_result $\& \mathrm{ct}=$ result $\&$ resnum $=1 \& \mathrm{ved}=0 C C s Q 6 \mathrm{AEwAA} \# \mathrm{v}=$ onepage $\& \mathrm{q}=\mathrm{Com}$ unidad $\% 20 y \% 20$ catequesis $\% 20$ de $\% 2$ Hungs $\& f=$ false

Lévy, P. 2007. Cibercultura, la cultura de sociedad digital. Barcelona: Anthropos, Universidad Autónoma Metropolitana. Extraído el 8 de Mayo de 2011 desde: http:// books.google.com/books?id=bn7Vf5dvbbYc\&pg=PA70\&dq=que+es+ciberespacio\&h I=es\&ei=E7BOTf7bAsfPrQf98KnZBg\&sa=X\&oi=book_result\&ct=result\&resnum=3\&v ed=oCDMQ6AEwAg\#v=onepage $\& q=q u e \% 20 e s \% 20$ ciberespacio $\& f=$ false 
Levy, P. 1999. ¿Qué es la virtualización? Lo actual y lo virtual. Editorial Paidos Ibérica S.A Extraído el 24 de Abril de 2011 desde: http://dintev.univalle.edu.co/pierrelevy/ documentos/pierre/QueEsLaVirtualizacion_PierreLevy.pdf

Mas, O. Jurado, P., Ruiz, C., Ferrández, E., Navío, A., Sanahuja, J.M. yTejada, J. 2006. Las comunidades virtuales de aprendizaje. Current Developments in TechnologyAssisted Education. 1462- 1466. Extraído el 21 de Abril de 2011 desde: http://es.scribd. com/doc/30097315/Comunidades-Virtuales-de-aprendizaje

Parra, A. 2003. Textos, contextos y pretextos. Teología fundamental. Bogotá: Pontificia Universidad Javeriana.

PUJ. Acuerdo No. 461 Plan de Desarrollo Tecnología El consejo directivo de la Pontificia Universidad Javeriana.

Unigarro G. M.A. 2004. Educación virtual, encuentro formativo en el ciberespacio. Bucaramanga: Unab. Extraído el 9 de Mayo de 2011 desde: http://books.google.com/ books?id=C03hWjUL9OAC\&pg=PA46\&dq=la+educacion+y+el+ciberespacio\&hl=es\&e $\mathrm{i}=\mathrm{E} 7 \mathrm{NOTY} 3 \mathrm{SBYrrrQf5}$ psXaBg\&sa=X\&oi=book_result\&ct=result\&resnum $=2 \&$ ved $=0 \mathrm{CC}$ 8Q6AEwAQ\#v=onepage\&q\&f=false

Yenes, P. 2000. Tecnologías de la información. Diccionario de la gestión del conocimiento e informática. Madrid: Fundación Dintel.

Zanotto, L. 2006. Profundizando y madurando nuestra fe. Londres: Editores en los campos. Extraído el 15 de Mayo de 2011 desde: http://books.google.com/books?id= RFShm6LvWc4C\&printsec=frontcover\&dq=Profundizando $+y+$ madurando+nuestra $+f$ $\mathrm{e}+\mathrm{de}+$ Zanotto $\& \mathrm{hl}=\mathrm{es} \& \mathrm{ei}=\mathrm{UoTjTdm} 8 \mathrm{NcaHrAftxbGdBg \& sa=X \& oi=book}$ result\&ct=res ult\&resnum=1\&ved=oCCkQ6AEwAA\#v=onepage $\& q \& f=$ false 